\author{
Aleksandra Rogowska \\ Opole University of Technology \\ Faculty of Physical Education and Physiotherapy \\ e-mail: a.rogowska@po.opole.pl
}

\title{
What can synaesthesia tell us about our minds?
}

\begin{abstract}
Synaesthesia is considered here as a cognitive phenomenon in the context of developmental, neuropathological and linguistic perspectives. Developmental synaesthesia seems to arise as an effect of interplay between genotype and phenotype, during the implicit learning process in childhood, in those individuals who possess an inborn susceptibility to it. Some connections between synaesthesia and extraordinary experiences, brain restructuration and pain, are examined. Acquired types of synaesthesia may be related to sensory deprivation. The somatosensory cortex may be significant for cognitive synaesthesia, with especial importance placed on a mirror system. It is suggested here that synaesthesia might play a compensatory role during the sensorimotor stage of development. Linguistic-colour synaesthesia seems to be an abstract type of association that may characterize people with a hypersensitive colour perceptual system. In the present view synaesthesia may be seen as an effect of some deficiency that concerns double integrative processes.
\end{abstract}

Keywords: synaesthesia; synaesthesia; developmental synaesthesia; acquired synaesthesia; temporary synaesthesia; linguistic-colour synaesthesia; abstract associations; implicit learning; somatosensory cortex.

\section{Implicit learning and developmental synaesthesia}

Synaesthesia does not seem to be so extraordinary as was previously assumed. Instead, numerous studies imply rather that synaesthesia occurs in many ways which are similar to common associations (e.g. Elias et al. 2003; Mulvenna and Walsh 2006; Cohen Kadosh et al. 2009a). For example, 
both synaesthetes and non-synaesthetes can associate high pitch sounds with bright colours, and low tones with dark colours (Marks 1989; Ward et al. 2006; Spence 2011). Also, many non-synaesthetes can link spatial forms with days of the week or months in a similar manner to time-space synaesthetes (Makioka 2009; Brang et al. 2010).

Synaesthesia may lie at the high end of a continuous spectrum of crossmodal connections (Martino and Marks 2001; Spence 2011; Cohen Kadosh and Henik 2007; Bien et al. 2012; Rogowska 2011, 2014). Martino and Marks (2001) suggest that weak synaesthesia represents common crossmodal associations dependent on individual experience and implicit knowledge about interactions between concrete objects (Marks et al. 1987). The perceptual knowledge about objects and events is represented in terms of locations in a multi-dimensional and multi-modal space. Strong synaesthesia refers to idiosyncratically more abstract associations (Rogowska 2014). However, there remains a question: is there a common mechanism that explains synaesthetic and nonsynaesthetic associations? What are the differences and connections between synaesthetic and common linguisticcolour associations?

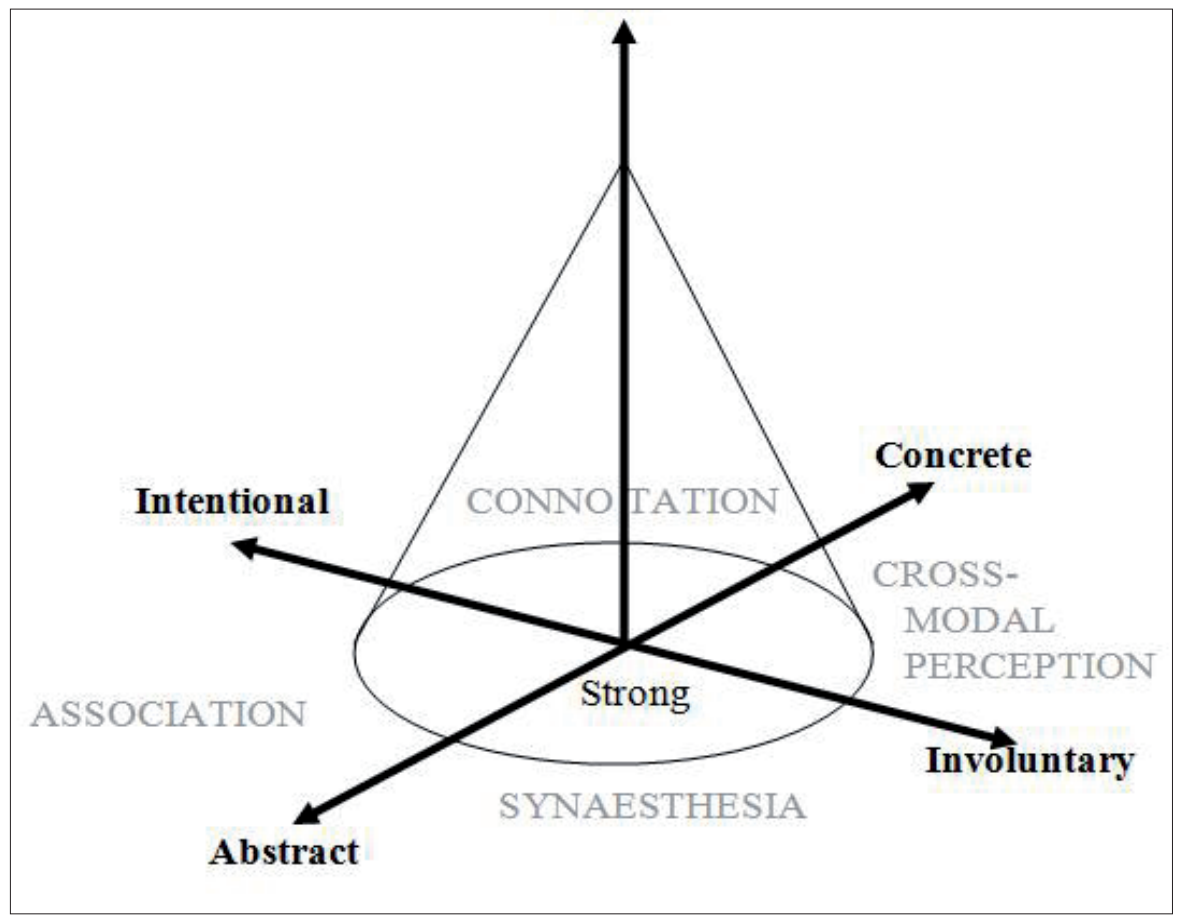

Figure 1. Hypothetical model of linguistic-colour associations 
There is some evidence (Hubbard 1996; Simner et al. 2005; Cohen Kadosh et al. 2007) that synaesthetes do not differ significantly from nonsynaesthetes in terms of the tendency to associate colours with linguistic inducers (e.g. letters of alphabet, numbers, words). Research on a random sample showed that synaesthetes are not easy to distinguish from nonsynaesthetes on the basis of measurement of test-retest consistency (Simner et al. 2006; Brang et al. 2010). A recent study (Rogowska 2014) implies rather that linguistic-colour synaesthesia is an extremely strong involuntary and abstract form of association, whereas nonsynaesthetic associations are more intentional and concrete (see Figure 1). The concrete-abstract dimension refers to the type of associations, whereas the weak-strong dimension applies to the quantity (strength) of these associations.

Moreover, in a study based on consistency of linguistic-colour associations, continuity and normal distribution for 161 participants were demonstrated (Rogowska 2014). Synaesthetic (abstract) linguistic-colour associations could be just as intense and consistent over time as concrete connotations (e.g. connections between fruits and their colours). There were neither qualitative nor quantitative differences between synaesthetes and non-synaesthetes in common concrete associations, such as fruits, months and seasons of the year. For example, lemon was associated with a yellow colour by all participants (Rogowska 2014). These results seem to suggest that synaesthesia, as well as cross-modal perception, may be a kind of implicit knowledge. A concrete word-colour association refers to the colour of an object that denotes the word in the closer nodes in the semantic network (Collins and Quillian 1969) and can be described as a typical, conventional, unoriginal and common association, derived from the learning process of enculturation.

The question is whether synaesthetic colour associations with abstract concepts (such as forenames, days of the week, numbers, letters of the alphabet) share the same rules. For example, what joins the synaesthetic colour(s) to non-synaesthetic colour association(s) for the forename category? A common non-synaesthetic association refers to colours that characterize a concrete familiar person with the given forename (e.g., colour of her/his eyes, hair), as is shown in Figure 2. Synaesthetic associations are usually related to abstract writing forms of the forename and the colour of this word may be affected by the first or dominant letter with graphemecolour synaesthetes. However, interaction between concrete and abstract colour association also seems possible.

Barnett et al. (2009) examined whether synaesthetic colour is consistently induced by grapheme or phoneme form or word meaning in bilingual and 
trilingual linguistic-colour synaesthetes. They found that induced colours to numbers, letters and days of the week were more related to the visual structure of the word across languages, whereas colours to months of the year were more related to higher levels of semantic information (i.e. word meaning) and were more consistent across languages than were colours to numbers or days of the week. The effect of inducing stimuli was also associated with the age of acquisition of additional languages.

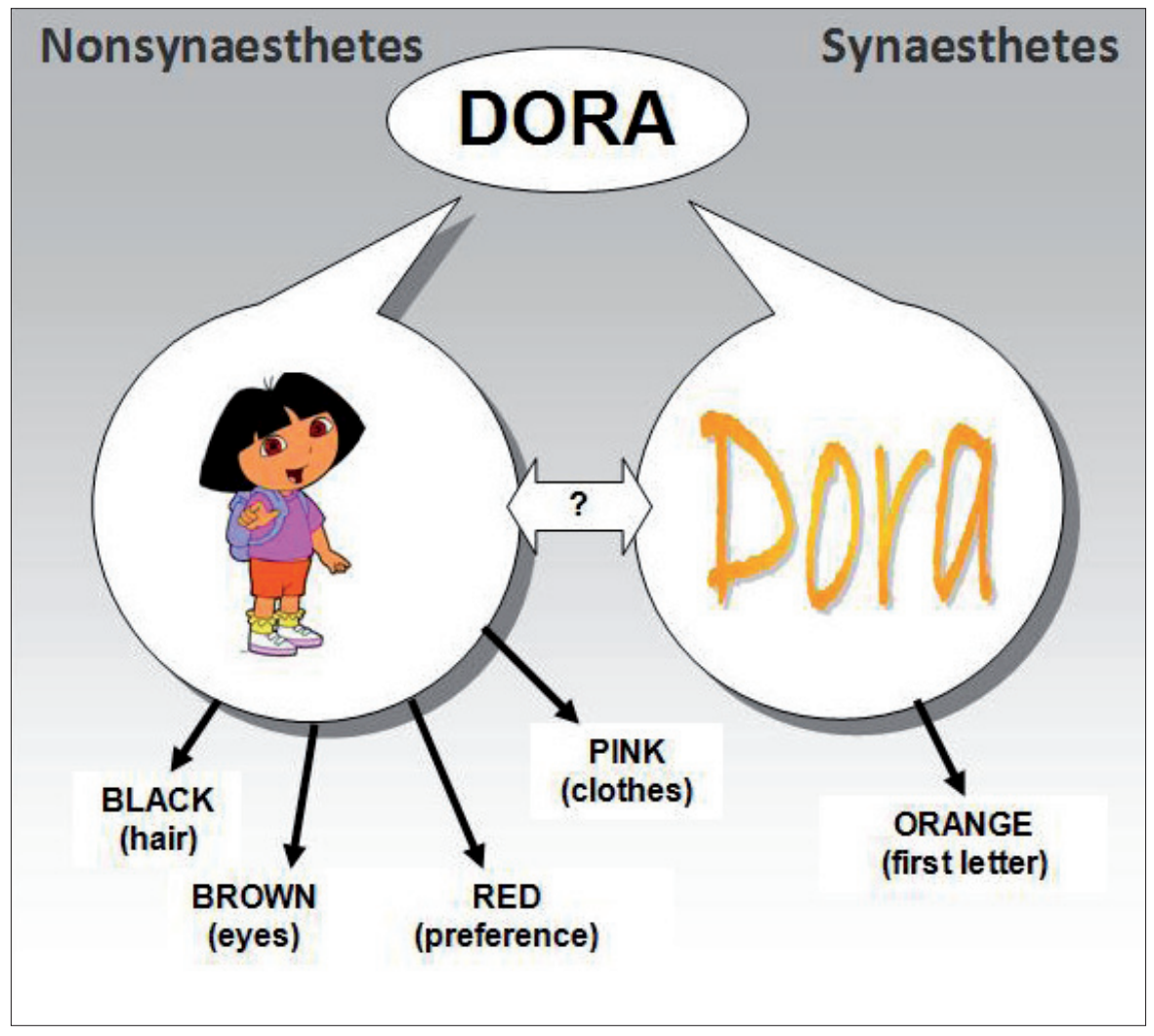

Figure 2. Typical forename-colour associations in nonsynaesthetes (on the left; association with concrete traits of "Dora") and synaesthetes (on the right; association with an abstract feature of graphemes) $)^{9}$

Grapheme-colour synaesthesia is connected to the knowledge of culturally acquired symbols and concepts (Nicolić 2009). A recent study

9 The clip art of Dora was accessed online from: http://www.disneyclips.com/imagesnewb6/dora.html (DOA: 25 January 2013); reprinted with permission of the Nick Jr. MTV. 
(Brang et al. 2011) implies that more similarly shaped graphemes correspond to more similar synaesthetic colours. Moreover, the results of current research (Watson et al. 2012) proved that synaesthetic associations are acquired as the alphabet is learned, with associations involving letter shape, ordinality, and frequency being made independently and idiosyncratically. These synaesthetic associations operate on principles common to many aspects of human cognition, as suggested by Watson et al. (2012).

Rich et al. (2005) have speculated that the learning of sequences during an early critical period may determine the particular pattern of lexical-colour links, and that this pattern can then be generalized to other words. Indeed, current research confirmed that grapheme-colour synaesthetic associations could be transferred and generalized into newly learned graphemes (Mroczko et al. 2009; Jürgens and Nicolić 2012; Asano and Yokosawa 2012). Moreover, there is evidence that synaesthesia is learned by showing that the frequency of everyday language implicitly modulates the synaesthetic experience (Cohen Kadosh et al. 2009b).

Witthof and Winaver (2006) demonstrated that the particular colours seen by a grapheme-colour synaesthete were learned from a set of refrigerator magnets. Furthermore, synaesthesia transferred into the Cyrillic script in a systematic way showed that colours induced by Cyrillic letters were determined by their visual or phonetic similarity to English letters. In the light of these findings, it seems appropriate to assume that synaesthesia arises as an effect of interplay between genotype and phenotype. Current data (Witthof and Winaver 2013) implies that learned synaesthesia may occur more commonly than was previously assumed, since 11 color-grapheme synaesthetes had startlingly similar colour-grapheme pairings traceable to childhood toys containing coloured letters. Witthof and Winaver (2013) argued that synaesthesia may be understood "as the automatic retrieval of highly specific mnemonic associations, in which perceptual contents are brought to mind in a manner akin to mental imagery or the perceptualreinstatement effects found in memory studies" (p. 258).

Synaesthesia could be an effect of an implicit learning process in those individuals who possess an inborn susceptibility to it. Indeed, the idiosyncratic character of synaesthetic experiences and the wide range of various types of synaesthesia (Day 2005; Novich et al. 2011) seem to confirm this assumption. However, the normal distribution of linguisticcolour associations (Rogowska 2007, 2014) suggests that synaesthesia may be determined by a nearly infinite number of factors beside the learning process (Makioka 2009; Cohen Kadosh et al. 2009a; Mroczko et al. 2009), such as cross-modal interactions (Marks 1975; Evans and Treisman 2010), 
metaphor-based connotations (Keith 2009; Seitz 2005), cognitive processing level (Ramachandran and Hubbard 2001; Ward et al. 2007), emotion (Ward 2004; Hochel et al. 2009), or an unknown number of cognitive skills, personality traits and environmental variables. Perhaps every synaesthete shows an idiosyncratic pattern of synaesthetic associations, which may be some kind of record of a person's unique interaction between individual traits and environment (like a photo in our family album). However, further research is needed on these issues in the future.

\section{Sensory deprivation and pathological synaesthesia}

\subsection{Synaesthesia and extraordinary experiences}

Synaesthesia seems to be related to pathological states of mind. Strong synaesthetic projections seem similar to hallucinations that are produced in cases of schizophrenia or caused by hallucinogenic drugs. Cytowic $(1997,2002)$ has suggested that synaesthetes seem more prone to unusual experiences, such as déjà vu, clairvoyance, precognitive dreams and a sense of portentousness. Indeed, some studies imply that synaesthesia is related to absorption, aura-vision, lucid-dreams and both mystical and out-of body experiences, which are also connected with dissociation and schizophrenia (e.g. Glicksohn et al. 1992, 2001; Thalbourne et al. 2001; Zingrone et al. 2009; Studerus et al. 2012). There is also some evidence that synaesthesia is related to sensory deprivation during extraordinary states of mind (Walsh 2005; Terhune 2009; Brang and Ramachandran 2008).

For example, Terhune (2009) has examined the relationships between hypnagogic imagery, unusual sleep experiences, weak synaesthesia and self-reported out of body experiences (OBEs). These studies revealed that the measure of weak synaesthesia (a seven-item true/false synaesthesia scale adapted from the Tellegen Absorption Scale) was the stronger predictor of visual types of OBEs. Terhune (2009) proposed a hypothesis that visual content may appear during both synaesthetic out of body experiences "through a process of cognitive dedifferentiation in which visual hallucinations are derived from available non-visual sensory cues and that such dedifferentiation is made possible through an underlying characteristic hyperconnectivity of cortical structures regulating vestibular and visual representations of the body and those responsible for the rotation of environmental objects" (p. 236).

The survey studies (Zingrone et al. 2009) showed that aura vision is related to synaesthesia-like experiences, mystical experiences and discrete 
psychic experiences (dissociation, absorption and depersonalization). In another study (Thalbourne et al. 2001), correlation has been found between synaesthesia and transliminal experiences. Transliminality reflects individual differences in the threshold at which unconscious processes or external stimuli enter into a person's consciousness (Fleck et al. 2008). Individuals who are high in transliminality possess characteristics such as magical ideation, belief in the paranormal, and creative personality traits, and also report the occurrence of manic or mystic experiences. Probably, in people revealing high transliminality, there is a shortage of cognitive mechanisms suppressing unrelated information at the consciousness level, due to which conscious and unconscious information processing is not regulated and the number of neural connections in the brain is increasing.

It has also been found that syncretic perception is related to synaesthesia, pseudo-hallucinatory imagination and absorption (Glicksohn et al. 1992; Studerus et al. 2012). In syncretic perception, sensory modalities are not diversified in cognitive terms and information processing takes place on a low, "primitive" level, which is typical for schizophrenics and artists (Glicksohn et al. 2001). Synaesthesia seems be associated with sensory deprivation during unusual states of consciousness. Walsh (2005) showed, by using a questionnaire survey, that in group of 49 Buddhist meditators, synaesthesia was experienced in 35\% of meditation retreatants, in $63 \%$ of a group of regular meditators and in $86 \%$ of advanced teachers.

Moreover, there is evidence that synaesthesia may be evoked or obstructed by psychoactive substances (Sinke et al. 2012; Ahmadi et al. 2011; Cytowic 2002; Brang and Ramachandran 2008), posthyponotic suggestions (Cohen Kadosh et al. 2009a; Terhune et al. 2010) or suggestive sensations (Biocca et al. 2001; Schaefer et al. 2006). Narcotic synaesthesia is encountered in substance-induced psychedelic states, such as with LSD (Ramachandran and Hubbard 2001) or dimethyltryptamine (DMT; Luke 2011). Amazonian cultures consume DMT as the primary psychoactive in ayahuasca, a shamanistic brew used for divinatory and healing purposes, such as synaesthesia, ostensible extra-dimensional percepts, out-of-body experiences, psi experiences and encounters with discarnate entities (Luke 2011; Shanon 2003). Brang and Ramachandran (2008) proposed that narcotic synaesthesia may be related to the activity of serotonin S2a receptors, which can be modulated by various drugs (or indeed by meditation).

A recent study (Studerus et al. 2010) revealed that altered states of consciousness induced by psilocybin and ketamine, such as experience of unity, spiritual experience, blissful state, insightfulness, disembodiment, impaired control and cognition or changed meaning of control, were correlated 
with audio-visual synaesthetic experiences. Audio-visual synaesthetic experiences were also related to absorption, emotional excitability, drug dose and alcohol consumption in healthy volunteers after psilocybin intake (Studerus et al. 2012).

A considerable similarity has also been revealed between abstract shapes in synaesthesias and hallucinations (Betancourt 2007). Furthermore, excitability disorders in the temporal area of cortex occur in the case of experiencing a déjà vu /epilepsy phenomenon or hallucinations in schizophrenic patients (Bancaud et al. 2004; Saugstad 2003; Jardri et al. 2009). Similar location is typical for temporary synaesthesia induced by epilepsy or ingestion of psychoactive drugs in people who normally do not reveal it (Kafka 1997; Jacome and Gumnit 1997; Jacome 2011; Sinke et al. 2012). Also, constitutional synaesthesia activates the same areas in the brain which are evoked by hallucinations in paranoid schizophrenia or in Charles Bonnet syndrome (Cohen Kadosh and Henik 2007).

Moreover, a study by Sarhan et al. (2008) implies that digit-colour synaesthetic-like experiences might be used as markers of schizophrenia. These findings may indicate a possible tendency for local cross-activation in the posterior parietal lobe in the context of both schizophrenia (due to aberrant connectivity) and synaesthesia (Esterman et al. 2006; Hubbard and Ramachandran 2005). Sarhan et al. (2008) indicates that there is evidence for a significant local increase in white matter connectivity in subjects with hallucinations compared to normal participants (Hubl et al. 2004). An understanding of synaesthesia could therefore contribute to explanations of cognitive disorders (Cohen Kadosh and Henik 2007).

Studies by Thornton-Wells et al. (2010) have examined brain responses to musical and other types of auditory stimuli in young adults with Williams syndrome (WS) and typically-developing controls, by using a functional MRI. Similar to synaesthesia-like experiences, the WS group exhibited unforeseen activations of the visual cortex to musical stimuli. Results of these studies seem to have implications for cross-modal sensory processing in typical and atypical neurodevelopment. Synaesthesia may be related to extraordinary inter-sensory configuration within the brain. Synaesthetes may have structurally or functionally different brains from non-synaesthetes. Thus, examination of extraordinary brain organization in psychopathological dysfunctions may be helpful towards explanations of both synaesthesia and cognitive disorders. 


\subsection{Synaesthesia and brain restructuration}

Synaesthesia may occur during adulthood as a result of biochemical brain changes, neurological dysfunctions, or permanent nervous system damage as a result of an accident or disease such as cancer, multiple sclerosis, or migraines (Afra et al. 2009; Niccolai et al. 2012; Altstadhaug and Benjaminsen 2010; Jacome 1999; Podoll and Robinson 2002; Rao et al. 2007; Ro et al. 2007; Villemure et al. 2006; Armel and Ramachandran 1999; Nold 1997). For example, in the single case-study described by Jacome (1999), a 54-year-old person had involuntary visions of small objects (Liliputian hallucinations) induced by musical sounds and sounds from the surroundings (e.g., an alarm clock ringing, a clock ticking, a dog barking) caused by damage of myelinic fibres in the temporal area of cortex as a result of multiple sclerosis (MS) development. If damage of a myelinic sheath led in the above case to double impressions, we can consider a similar source of synaesthesia origination in early childhood when the process of myelinisation of nerve fibres develops.

Another pathological aspect of synaesthesia seems to be related to brain injuries caused by accidents, cancerous changes or loss of perceptual abilities in any of the senses, as a result of progressive pathological changes in receptors or neural tissue. Frequent appearance of acquired synaesthesia occurs in people who lost their visual ability, which is probably a case of compensation of lost visual perception (Nold 1997). The case of visualtactile synaesthesia, acquired at the age of 42 , after a complete loss of visual ability (retinitis pigmentosa), indicates that neural connections in an adult brain may be modified by centrifugal signals of feedback, causing forwarding of neural impulses simultaneously to the somatosensory cortex and to the extrastriate cortex, forming synaesthetic visual projections (Armel and Ramachandran 1999). Steven et al. (2006) demonstrated by using fMRI that linguistic-colour synesthesia may be truly a perceptual experience in a man with retinitis pigmentosa, who has been completely blind for 10 years.

Proulx and Stoerig (2006) suggest that synaesthesia might arise from sensory deprivation that very often induces hallucinations in the modality deprived of its input. Indeed, linguistic-colour synaesthesia is often reported in blind people (Jacobs et al. 1981; Armel and Ramachandran 1999; Afra et al. 2009). According to Proulx and Stoerig (2006), synaesthesia may be better understood by explanation of sensory substitution phenomenon that "attempts to substitute a sensory modality that a person has lost by transforming the information it provided so that it can be accessed through another, intact sensory modality" (p.135). For example, by using the 
vOICe, visual information can be provided to blind users through sensory substitution devices such as those which convert images into sound. Recent research (Proulx 2010; Ward and Meijer 2010) has revealed that, in some cases, the blind expert users of the sensory substitution devices may develop an artificial type of acquired synaesthesia, in which visual experiences are evoked by sounds. Moreover, sensorimotor learning may facilitate and perhaps even be required to develop expertise in the use of multimodal information (Proulx 2010).

Ward (2007) described a single-case study of acquired auditorytactile synaesthesia. Beauchamp and Ro (2008) revealed that neural plasticity induced by a stroke can mediate positive outcomes, such as recovery of a function, but can also result in the formation of abnormal connections between sensory modalities that are normally separate (in the secondary somatosensory cortex), and that sound-touch synaesthesia can be caused by inappropriate connections between nearby cortical territories. Unfortunately, explanations of different kinds and types of synaesthesia may depend on distinct neurocognitive and psychological mechanisms (Cohen Kadosh and Terhune 2012; Eagleman 2012; Sinke et al. 2012; Afra et al. 2009). However, one can assume that sensory deprivation, such as an effect of disorder within the nervous system, may contribute (among other factors) to acquired synaesthesia. The question is whether sensory deprivation is a universal factor for all other types of synaesthesia (including constitutional/developmental).

\subsection{Synaesthesia and pain}

An anomalous cortical processing underlying synaesthesia may be related to pain. In the case report described by Alstadhaug and Benjaminsen (2010), a 40-year-old woman with a cyclic mood disorder had suffered from migraine, with several episodes of visual-taste synaesthetic experiences during the visual aura phase. Also, Podoll and Robinson (2002) reported occurrence of auditory-visual acquired synaesthesia in a 66-year-old art teacher who suffered from attacks of basilar migraine with aura. For this woman, an alarm clock could induce on several occasions a coloured optical pattern in the centre of her field of vision, pulsing in correspondence with the sound. Podoll and Robinson (2002) mentioned two other case reports of auditory-visual synaesthetic episodes co-occurring with migraine. Furthermore, Rogowska (2014) provided evidence, by using self-report questionnaire methods, that linguistic-colour synaesthetes significantly experienced headache or migraine more frequently than non-synaesthetes. 
Among 572 synaesthetes recorded by Day (2005), 36 (6.3\%) reported pain-colour synaesthesia. One of the most famous synaesthetes, Carol Steen, had experienced coloured pain during an acupuncture session (Steen, 2001). She had painted her synaesthetic visions in several abstract oils. Hornik (2001) described one of them as "a green slash arcing through a field of red" and another one as "a tiny red triangle drifts off into the distance on a sea of bright blue". The cases of pain colour synaesthesia seem to imply that the state of pathological pain may influence metabolic changes in the brain, leading to synaesthesia.

Indeed, numerous studies (e.g. Ramachandran et al. 1995; Ramachandran and Rogers-Ramachandran, 1996, 2000; Mon-Williams et al. 1997) demonstrated phantom synaesthesia cases, in which a patient could feel sensations in an amputated arm, or could experience pain in a phantom limb, during visual feedback by using of a special mirror box ("virtual reality box"). In these cases, reorganisation within the somatosensory cortex seems to result in synaesthesia.

A recent study (Carruthers et al. 2012) examines the relationships between irritable bowel syndrome (IBS) and grapheme-colour and musiccolour (shape) synaesthesia. Irritable bowel syndrome can be characterized by chronic abdominal pain, discomfort, bloating, and alteration of bowel habits. The prevalence of synaesthetes among the IBS patients was significantly higher (13\% of 200$)$ in comparison to the healthy individuals ( $3 \%$ of 200$)$. Moreover, the intensity of the grapheme-color synaesthetic or pseudosynaesthetic experiences was significantly greater in the IBS individuals than in the healthy volunteers. It seems to imply that the increased connectivity between different areas of the brain as demonstrated in synaesthetes might also predispose them to perceptual distortions contributing to IBS.

Mirror-touch synaesthetes experience tactile sensations on their own body when observing someone else being touched (Banissy and Ward 2007; Banissy et al. 2009a). Blakemore et al. (2005) found that visual perception of touch elicits conscious tactile experiences in the somatosensory cortex of the perceiver. However, in a mirror-touch synaesthete female subject (C), the mirror system for touch in the somatosensory cortex was significantly higher, above the threshold for conscious tactile perception, when she observed touch. Pain synaesthetes experienced an observed or imaginary pain as if it was their own (Fitzgibbon et al. 2010). It seems to be no coincidence that synaesthesia could be formed in either mirror-touch or pain types and that synaesthesia is related to touch and pain in many ways. The somatosensory cortex might play a key role in cognitive synaesthesia (higher type), with especial importance of a mirror system. 


\section{Integration process in cognitive synaesthesia}

According to Piaget's theory (1952), during the sensorimotor stage (the first two years of life), infants explore relationships between their bodies and the environment, on the basis of informal experimentation with objects, by using multi-sensory perceptions (e.g., seeing, touching, smelling, tasting-sucking) and motor activities. In this period, a child creates mental representations of objects by dominant participation of sensory integration processes, which is a key for forming symbolic thought in later stages of intelligence development. Due to evolving language and cognitive abilities in memory, imagination and attention, concrete operations are replaced by abstract thinking in the next years of life.

Synaesthesia might play a compensatory role (Rogowska 2011) during the sensorimotor stage of development, if one of the modalities is impaired, functions poorly or as a result of sensory deprivation, in a similar way to other types of synaesthesia (e.g., phantom, artificial, virtual, narcotic, or posthypnotic synaesthesia). A current study (Rogowska 2014) seems to imply that linguistic-colour synaesthesia is related to some kinds of deficits in the somatosensory association cortex. This region of the brain seems very important for synaesthesia in general. Cortical injury in the somatosensory system can lead to loss of thermal sensation and inability to discriminate pain, aura vision during migraine (that involving thermal and painful sensations), and sensory Jacksonian seizure (involving an abnormal, localizable, cutaneous sensation but which does not have apparent stimulus). Some investigations mentioned above imply that synaesthesia also may be related to pain sensations in many ways.

\subsection{Linguistic-colour synaesthesia}

Language in humans might be considered as a kind of "sixth sense". Similar to other senses, language demonstrates a limited range of stimulation (i.e., native language and selected foreign languages, in some cases). Moreover, language demonstrates a hierarchical and modular structure, such as other senses. However, because language concerns a higher level "semantic sense", it operates integrated information derived from the other five senses through a system of integration itself (see Figure 3). Mirror systems in the somatosensory cortex might play a key role of a language's receptor.

On the base of self-observation, I have formed the view that when familiar verbal information is detected from an external world during 
listening to speech, the articulatory system repeats it automatically by using "inner voice", almost at the same time, like an echo. Most likely it may concern people for whom spoken language was a first step in the development of communication process (Auditory Language First, ALF). If someone starts to learn a language by using primarily iconic language (Iconic Language First, ILF), native written language (Written Language First, WLF), Braille writing system (Braille System First, BSF) or sign language (Sign Language First, SLF), he or she may use visual, tactile or motor modules for echoic repetition system, respectively.

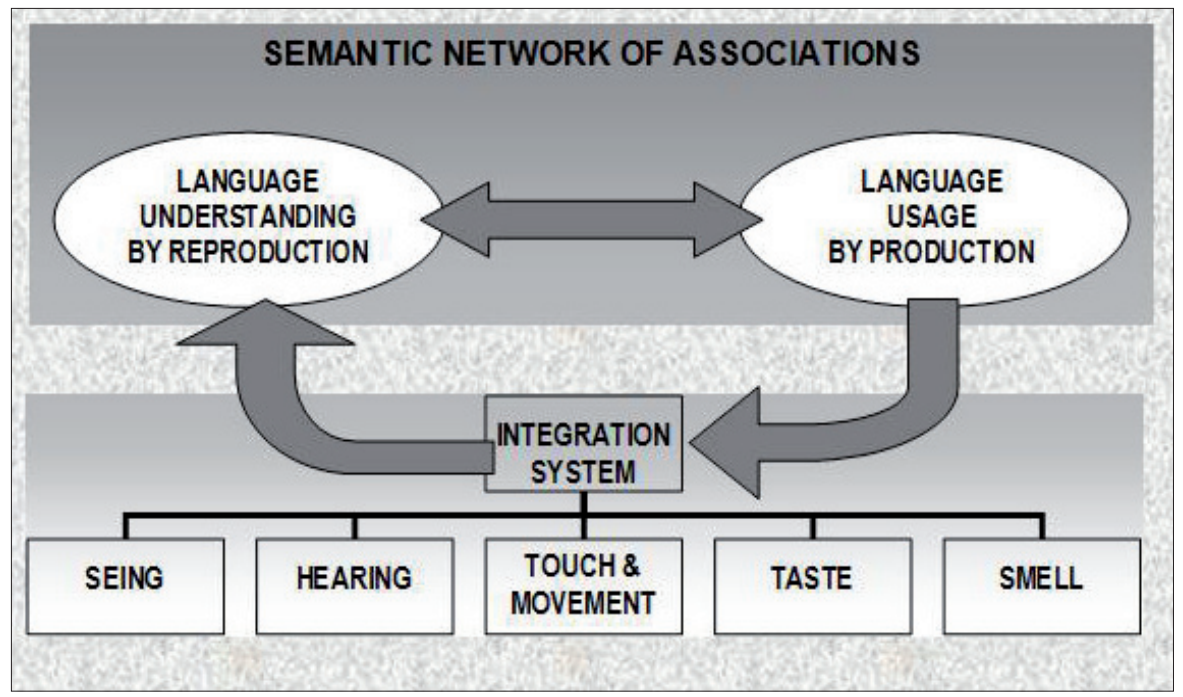

Figure 3. Hypothetical model of integration during language processing

Automatic verbal echoes may be easy to identify in ALF when a novice tries to understand a foreign language while listening to audio-records. If the novice is unable to repeat the word by using his/her own inner echo voice, it is impossible to form an understanding. According to self-observations, when a person generates sensible statements (by using either an articulatory system or inner voice), he or she is unable to simultaneously understand another person's speech. Comparative research among various groups of adults (i.e., ALF, ILF, WLF, BSF and SLF) using neurocognitive methods is necessary for verification of these assumption and self-observations.

It is suggested here that input into the conscious semantic level is only by the "gate" of echoic repeat system of speech, by using either inner voice, or other sensory information, respectively to first (or maybe dominant?) language. Conscious perception (e.g., detecting, recoding and identification) 
of external verbal stimulation is only possible by its echoic reproduction, through activation of mirror systems, most likely. Similarly, production of speech is a way to express conscious thinking processes. In this context, language (which can be widely interpreted to include speech, music and art) might be an equivalent to consciousness.

Understanding another person's speech presented auditorally is possible by automatic inner echo in ALF, which is most likely related to mirror systems within the somatosensory cortex. This inner echo can start a chain of associations within a semantic network that provides for recognizing words, uncovering meaning and understanding. Also, a reading of visually presented words relies on generating an inner voice in ALF, involved in the articulatory system and somatosensory cortex as well. Analogically, congenitally deaf person may use visual (in ILF), motor (in SLF), or tactile (in BLF) echoic representations of language that are associated with mirror system. The above hypothesis requires research.

In correspondence with Baddeley and Hitch's (1974) model of working memory, linguistic-colour synaesthesia may be the result of association between the phonological loop (PL) and the visuo-spatial sketchpad. Moreover, synaesthetes might use the visuo-spatial sketchpad for constructing a coloured image of words. Perhaps, synaesthetes demonstrate both slave systems simultanously; whereas, in most people, these two slave systems rather function separately or with significant dominance of either one or the other. Thus, the central executive system that is responsible for the control and regulation of cognitive processes in synaesthetes might be more capacious. It may be an effect of a higher degree of network density within the central module (somatosensory cortex).

According to the hyper-connectivity concept of grapheme-colour synaesthesia, there is a globally altered network architecture in the graphemecolour synaesthete's brain. Hänggi et al. (2011) have provided evidence that the globally altered structural network topology was reflected by reduced small-worldness, increased clustering, increased degree, and decreased betweenness centrality, and also by increased intramodular and intermodular connectivity of the fusiform gyrus (FuG) and intraparietal sulcus (IPS). This global hyperconnectivity may imply that there are numerous relationships between modalities in synaesthetes' brains (Bargary and Mitchell 2008; Hänggi et al. 2011).

Linguistic-colour synaesthesia might be related to specific brain organization that relies on a more symmetrical representation of language (instead of left hemispheric dominance) in one-sided women and lateralized men, and also in poor somatosensory system functioning, as revealed by 
a current study (Rogowska 2014). According to the compensatory hypothesis (Rogowska 2011), some deficit in touch perception may be compensated for by colour in linguistic-colour synaesthesia during the sensorimotor stage of development (Piaget 1954). Thus, detection of a specific configuration of neurocognitive features in vision, hearing, and motor control, as well as a language process, may be a key issue for explanation of linguistic types of synaesthesia.

Linguistic-colour synaesthesia seems to be an abstract type of association that may characterize people with a hyper-sensitive colour perceptual system (Yaro and Ward 2007; Brang et al. 2010; Banissy et al. 2009b). Generally, better functioning sensory modalities at a given stage of cognitive development may share in forming additional attributes for mental representation of either concrete objects or abstract concepts in synaesthesia. For example, if colour perception is better developed than other senses, it is likely that colour will be the synaesthetic concurrent. Indeed, there is some evidence (Banissy et al. 2009b; Rogowska 2014) that synaesthetes who have experienced concurrent colour sensation demonstrated enhanced colour sensitivity.

\subsection{Double integration}

Understanding how synaesthesia is mediated in the brain can help to understand normal processes of perceptual awareness and multisensory integration (van Leeuwen et al. 2010). Kallenbach et al. (2001) revealed, by using position emission tomography, that knowledge about the typical color, size, and sound of common objects and animals activated similar left temporal and frontal regions. These results indicated that the retrieval of perceptual semantic information activates not only a general semantic network, but also cortical areas specialized for the modality and attribute type of the knowledge retrieved. Recent findings (Chiou et al. 2013) suggest theories of the brain mechanisms of grapheme-colour synaesthesia need to incorporate a broader neural network underpinning multiple visual features, perceptual knowledge, and feature integration, rather than solely focusing on colour-sensitive areas. Chiou et al. (2013) found evidence that visual synaesthetic experience can involve multiple features forming object-like percepts and suggest that each feature can be selected by attention despite it being internally generated.

Perhaps synaesthesia is an effect of some deficiency that concerns integrative processes. Linguistic-colour synaesthesia occurs at the semantic level and is closely related to the significance of a stimulus. Perhaps there are 
doubled integrative processes: 1.) the first stage of integration concerns the sensory system and is based on physical features of multimodal information received from the external world; and 2.) the second stage of the integration process is related to language and refers to a semantic system that is based on meaning of information connected with other cognitive subsystems in the mind, such as sensory modalities, memory, imagination, attention and emotion (see Figure 4).

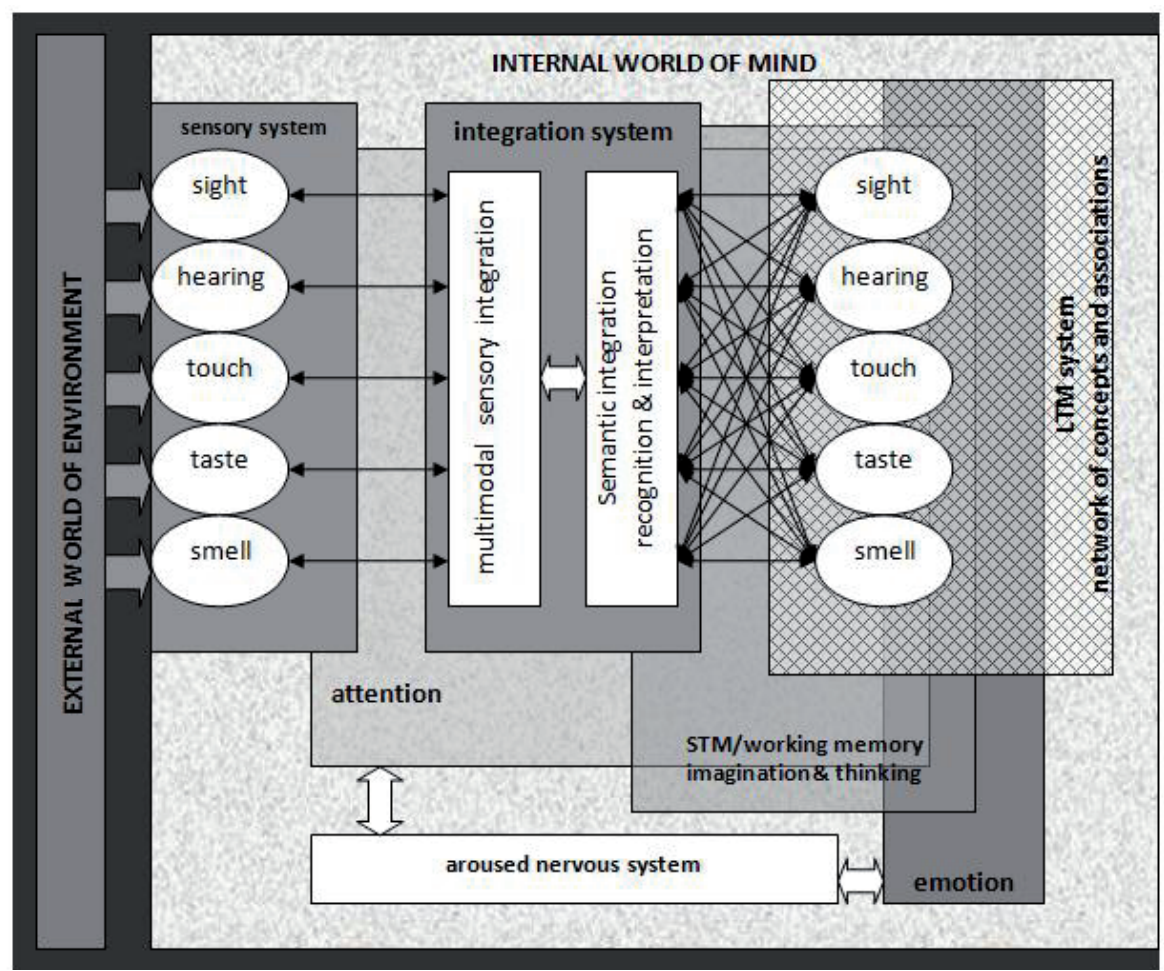

Figure 4. Hypothetical model of mind with a mirror integration system of information from the external and internal world

The first stage of multimodal integration of information reflects features of the external world, whereas the second stage reflects features of the internal world of mind. These two integrative systems functioning relatively independently of each other; however, they cooperate by creating a mental representation of the external world. Three subsystems (memory, attention, and emotions) seem to play an important role for processing the meaning of information; therefore, such sub-systems may be specifically interrelated. The first stage of integration may be more connected to sensory types 
of synaesthesia (lower synaesthetes, projectors), whereas the second stage of integration may concern more cognitive types of synaesthesia (higher synaesthetes, associators). Recent research (van Leeuwen et al. 2011) by using dynamic causal modeling for fMRI, seems confirmed this assumption. According to competing hypotheses, van Leeuwen et al. (2011) revealed that "V4 cross-activation during grapheme-color synaesthesia was induced via a bottom-up pathway (within fusiform gyrus) in projector synaesthetes, but via a top-down pathway (via parietal lobe) in associators" (p. 9879).

According to Piaget's (1954) developmental stages theory, the first stage of integration may correspond to assimilation processes, whereas the second stage with accommodation. The degree of fit between the first and second stages of integration increases the effectiveness of cognitive adaptation. If a great amount of information becomes deformed or missing (e.g., because of sensory deprivation) during this process of adjusting between the first and second level of integration (e.g. Strauss 1993), it may lead to a cognitive disorder such as with hallucinations, and acquired or narcotic synaesthesias (e.g., Sinke et al. 2012; Sarhan et al. 2008).

A multimodal representation, which is created during the first process of integration at the sensory level, consists of a large amount of information. Because of cognitive limitations, it is necessary to reflect an effect of this process into a compact and synthetic form, such as a symbol (e.g., concept, word, picture). This process of reduced information would occur at the second stage of integration, on the semantic level. A compact semantic representation contains the necessary number of features required to recognise an object and to use it in working memory. A concept, together with its attributes, knowledge about it (both episodic and semantic) and emotional component, are recorded in a semantic network within the long-term memory (LTM). The significance of a stimulus triggers a chain of associations, facilitating access to the elements recorded in memory that can be useful in information processing.

A hypothetical model of mind was proposed here for description and explanation of synaesthesia phenomenon. Discerning analysis and verification of these models exceeds the capacity of the present article. However, there are many questions for consideration in future research. For example, what is association between particular types of synaesthesia and automaticity of higher mental process (Bargh 2011)? What is the place of synaesthetic information within abstract concepts (such as numbers, days of the week, months of the year, etc.) due to the theory of simulation as a basic computational mechanism in the brain (Barsalou 2009)? How may the extending of linguistic types of synaesthesia (e.g., grapheme- 
colour, forename-colour, ordinal linguistic synaesthesia, etc.) be relevant for explaining language and other cognitive mechanisms according to neuroscientific principles (Pulvermüller 1999, 2001)? What may connect emotion with synaesthesia, in light of the somatic marker hypothesis (Damasio et al. 1991)?

It does not seem to be a coincidence that individual senses are represented in different regions of the cerebral cortex, forming particular modalities; also, that particular sensory receptors are arranged in distinct places of the body. This cluster-modular sensory organization may be most useful for the first integrative processes. Meunier et al. (2010) suggested that modularity of brain network organization might be related to the kind of unconscious/ specialized (or conscious/general) cognitive processing that it can support.

Hierarchical modular networks represent an economical way of embedding topologically complex systems in relatively low-dimensional physical space. Bassett et al. (2010) assumed that "artificial and biological information processing systems both may evolve to optimize a trade-off between physical cost and topological complexity, resulting in the emergence of homologous principles of economical, fractal and modular design across many different kinds of nervous and computational networks" (p. 1). As such, the best co-worked sub-modules are arranged together (and closer to each other) in higher-order clusters. For example, hearing and touch cooperate during language processing and sound recognition (e.g., sound causes body vibration). The study by Ro et al. (2009) implies that auditory information influences touch perception in highly systematic ways and suggests that similar coding mechanisms may underlie the processing of information from these different sensory modalities.

On the other hand, a semantic network may consist of an infinite number of links that decide about the present inducer-concurrent association. The mind contains an innumerable amount of recorded information; therefore, only an appropriate system of arrangement or cataloguing information in memory enables effective and easy access to the data and manipulation of information during identification/differentiation and thinking processes. In this view, synaesthesia most likely shows the idiosyncratic configuration of minds; how the mind forms internal representations of objects from the external world, and how it organises information within particular modalities. Proulx et al. (in press) suggested that investigations of auditory, visual and multisensory perceptual learning can have key benefits for the advancement of sensory substitution (sensory deprivation) in particular, and for an understanding of perceptual learning in general. It also has significant importance for the developing of our understanding of the brain in metamodal terms. Proulx 
(2010) found that synthetic mappings are required to generalize perceptual learning to new objects and environments, and ultimately to experience visual qualia. Moreover, sensorimotor experience may be required to facilitate learning, develop expertise, and to develop a form of synthetic synaesthesia.

If we all are some kind of synaesthete (Maurer 1997; Maurer and Mondloch 2005), every one of us may demonstrate a unique pattern of synaesthetic integration, like our epidermal ridges. This specific synaesthetic pattern may show an individual history of interaction between cognitive development and environment, between particular senses and concepts during integration process, and also between our cognitive abilities and dysfunctions in particular stages of development. This kind of information could be very useful for a cognitive, developmental, and clinical psychologist as well as for a neuroscientist. Show me your type of synaesthesia, and I will tell you what is in your mind!

\section{References}

Afra, Pegah, Michael Funke and Fumisuke Matsuo. 2009. "Acquired auditory-visual synesthesia: A window to early cross-modal sensory interactions." Psychology Research and Behavior Management 2: 31-37.

Ahmadi, Jamshid, Mitra Keshtkar and Saxby Pridmore. 2011. "Methamphetamine induced synesthesia: a case report." The American Journal on Addictions 20: 306.

Alstadhaug, Karl B. and Espen Benjaminsen. 2010. "Synesthesia and migraine: case report.” BMC Neurology 10: 121. DOI:10.1186/1471-2377-10-121.

Armel, Kathleen Carrie and Vilayanur S. Ramachandran. 1999. "Acquired synesthesia in retinitis pigmentosa." Neurocase 5:293-296.

Asano, Michiko and Kazuhiko Yokosawa. 2012. "Synesthetic colors for Japanese late acquired graphemes." Consciousness and Cognition 21: 983-993.

Baddeley, Alan D. and Graham J. L. Hitch. 1974. Working Memory. In Gordon $\mathrm{H}$. Bower (ed.), The psychology of learning and motivation: advances in research and theory (vol. 8), 47-89. New York: Academic Press.

Bancaud, J., F. Brunet-Bourgin, P. Chauvel and E. Halgren. 2004. "Anatomical origin of déjà $v u$ and vivid 'memories' in human temporal lobe epilepsy." Brain 117: 71-90.

Banissy, Michael J., Roi Cohen Kadosh, Gerrit W. Maus, Vincent Walsh and Jamie Ward. 2009a. "Prevalence, characteristics and a neurocognitive model of mirror-touch synaesthesia." Experimental Brain Research 198: 261-272.

Banissy, Michael J., Vincent Walsh and Jamie Ward. 2009b. "Enhanced sensory perception in synaesthesia." Experimental Brain Research 196: 565-571. 
Banissy, Michael J. and Jamie Ward. 2007. "Mirror-touch synaesthesia is linked with empathy." Nature Neuroscience 10: 815-816.

Bargary, Gary and Kevin J. Mitchell. 2008. "Synaesthesia and cortical connectivity." Trends in Neurosciences 31: 335-342.

Bargh, John A. 2011. "Unconscious thought theory and its discontents: a critique of the critiques." Social Cognition 29.6: 629-647.

Barnett. Kylie J., Joanne Feeney, Michael Gormley and Fiona N. Newell. F. N. 2009. "An exploratory study of linguistic-colour associations across languages in multilingual synaesthetes." The Quarterly Journal of Experimental Psychology 62.7: 1343-1355.

Barsalou, Lawrence W. 2009. "Simulation, situated conceptualization and prediction." Philosophical Transactions of the Royal Society B 364: 12811289. DOI:10.1098/rstb.2008.0319

Bassett, Danielle S., Daniel L. Greenfield, Andreas Meyer-Lindenberg, Daniel R. Weinberger, Simon W. Moore and Edward T. Bullmore. 2010. "Efficient physical embedding of topologically complex information processing networks in brains and computer circuits." PLoS Computational Biology 6: e1000748. DOI:10.1371/journal.pcbi.1000748.

Beauchamp, Michael S. and Tony Ro. 2008. "Neural substrates of sound-touch synesthesia after a thalamic lesion." The Journal of Neuroscience 28: 13696 -13702 .

Betancourt, Michael. 2007. "A taxonomy of abstract form using studies of synaesthesia and hallucinations."'Leonardo 40: 59-65.

Bien, Nina, Sanne ten Oever, Rainer Goebel and Alexander T. Sack. 2012. "The sound of size crossmodal binding in pitch-size synesthesia: A combined TMS, EEG and psychophysics study." NeuroImage 59: 663-672.

Biocca, Frank, Jin Kim and Yung Choi. 2001. "Visual touch in virtual environments: An exploratory study of presence, multimodal interfaces and cross-modal sensory illusions." Presence 10: 247-265.

Blakemore, S. -J., D. Bristow, G. Bird, C. Frith and J. Ward. 2005. "Somatosensory activations during the observation of touch and a case of vision-touch synaesthesia." Brain 128: 1571-1583.

Brang, David and Vilayanur S. Ramachandran. 2008. "Psychopharmacology of synaesthesia: The role of serotonin S2a receptor activation." Medical Hypotheses 70: 903-904.

Brang, David, Romke Rouw, Vilayanur S. Ramachandran and Seana Coulson. 2011. "Similarly shaped letters evoke similar colors in grapheme-color synesthesia." Neuropsychologia 49: 1355-1358.

Brang, David, Ursina Teuscher, Vilayanur S. Ramachandran and Seana Coulson. 2010. "Temporal sequences, synesthetic mappings and cultural biases: The geography of time." Consciousness and Cognition 19: 311-320.

Carruthers, Helen R., Vivien Miller, Nicholas Tarrier and Peter J. Whorwell. 2012. "Synesthesia, pseudo-synesthesia, and irritable bowel syndrome." Digestive Diseases and Sciences 57: 1629-1635. DOI: 10.1007/s10620-012-2054-2. 
Cohen Kadosh, Roi and Avishai Henik. 2007. "Can synaesthesia research inform cognitive science?" Trends in Cognitive Sciences 11: 177-184.

Cohen Kadosh, Roi, Avishai Henik, Andres Catena, Vincent Walsh and Luis J. Fuentes. 2009a. "Induced cross-modal synaesthetic experience without abnormal neuronal connections." Psychological Science 20: 258-265.

Cohen Kadosh, Roi, Avishai Henik and Vincent Walsh. 2007. "Small is bright and big is dark in synaesthesia." Current Biology 17: 834-835.

Cohen Kadosh, Roi, Avishai Henik and Vincent Walsh. 2009b. "Synaesthesia: learned or lost?" Developmental Science 12: 484-491.

Cohen Kadosh, Roi and Devin B. Terhune. 2012. "Redefining synaesthesia?" British Journal of Psychology 103: 20-23. DOI: 10.1111/j.2044-8295.2010.02003.x

Collins, Allan M. and M. Ross Quillian. 1969. "Retrieval time from semantic memory." Journal of Verbal Learning and Verbal Behavior 8: 240-247.

Cytowic, Richard E. 2002. Synaesthesia: A union of the senses (2nd ed.). New York: Springer-Verlag.

Cytowic, Richard E. 1997. Synaesthesia: Phenomenology and neuropsychology a review of current knowledge. In Simon Baron-Cohen and John E. Harrison (eds.), Synaesthesia: Classic and contemporary readings, 17-39. Oxford: Blackwell.

Damasio, Antonio R., Daniel Tranel and Hanna C. Damasio. 1991. Somatic markers and the guidance of behavior: theory and preliminary testing. In Harvey S. Levin, Howard M. Eisenberg and Arthur L. Benton (eds.), Frontal lobe function and dysfunction, 217-229. New York: Oxford University Press.

Day, Sean A. 2005. Some demographic and socio-cultural aspects of synesthesia. In Lynn C. Robertson and Noam Sagiv (eds.), Synesthesia: Perspectives from cognitive neuroscience, 11-33. New York: Oxford University Press.

Eagleman, David M. 2012. "Synaesthesia in its protean guises." British Journal of Psychology 103: 16-19. DOI: 10.1111/j.2044-8295.2011.02020.x

Elias, Lorin J., Deborah M. Saucier, Colleen Hardie and Gordon E. Sarty. 2003. "Dissociating semantic and perceptual components of synaesthesia: behavioural and functional neuroanatomical investigations." Cognitive Brain Research 16: 232-237.

Esterman, Michael, Timothy Verstynen, Richard B. Irvy and Lynn C. Robertson. 2006. "Coming unbound: disrupting automatic integration of synesthetic color and graphemes by transcranial magnetic stimulation of the right parietal lobe." Journal of Cognitive Neuroscience 18: 1570-1576.

Evans, Karla K. and Anne Treisman. 2010. "Natural cross-modal mappings between visual and auditory features." Journal of Vision 10: 1-12.

Fitzgibbon, Bernadette M., Melita J. Giummarra, Nellie Georgiou-Karistianis, Peter G. Enticott and John L. Bradshaw. 2010. "Shared pain: From empathy to synaesthesia". Neuroscience and Biobehavioral Reviews 34: 500-512.

Fleck, Jessica I., Deborah L. Green, Jennifer L. Stevenson, Lisa Payne, Edward M. Bowden, Mark Jung-Beeman and John Kounios. 2008. "The transliminal 
brain at rest: baseline EEG, unusual experiences, and access to unconscious mental activity." Cortex 44: 1353-1363. DOI: 10.1016/j.cortex.2007.08.024.

Glicksohn, Joseph, Ariel Alon, Ayala Perlmutter and Ruth Purisman. 2001. "Symbolic and syncretic cognition among schizophrenics and visual artists." Creativity Research Journal 13: 133-143.

Glicksohn, Joseph, Orna Salinger and Anat Roychman. 1992. “An exploratory study of syncretic experience: Eidetics, synaesthesia and absorption." Perception 21: 637-642.

Hänggi, Jürgen, Diana Wotruba and Lutz Jäncke. 2011. “Globally altered structural brain network topology in grapheme-colorsynesthesia." The Journal of Neuroscience 31: 5816 -5828.

Hochel, Matej, Emilio Gómez Milán, Jose Luis Mata Martín, A. González, Emilio Domínguez García, Francisco JoseTornayMejias and Jaime Vila Castellar. 2009. "Congruence or coherence? Emotional and physiological responses to colours in synaesthesia." European Journal of Cognitive Psychology 21: 703-723.

Hornik, Susan. (2001, Ferbuary 1). "For some, pain is orange." Smithsonian Magazine 31: 48-54.

Hubbard, Edward M. and Vilayanur S. Ramachandran. 2005. "Neurocognitive mechanisms of synesthesia." Neuron 48: 509-520.

Hubbard, Timothy L. 1996. "Synesthesia-like mappings of lightness, and melodic interval." The American Journal of Psychology 109: 219-228.

Hubl, Daniela, Thomas Koenig, Werner Strik, Andrea Federspiel, Roland Kreis, Chris Boesch, Stephan E. Maier, Gerhard Schroth, Karl Lovblad and Thomas Dierks. 2004. "Pathways that make voices: white matter changes in auditory hallucinations." Archives of General Psychiatry 61: 658-668.

Jacobs, Lawrence, Alice Karpik, Diana Bozian and Svend Gøthgen. 1981. "Auditoryvisual synesthesia: Sound-induced photisms." Archives of Neurology 38: 211216.

Jacome, Daniel E. 1999. "Volitional moncularliliputian visual hallucinations and synesthesia." European Neurology 42: 54-56.

Jacome, Daniel E. 2011. "Sound induced photisms in pontine and extrapontinemyelinolysis." Clinical Neurology and Neurosurgery 113(6):503505 DOI: 10.1016/j.clineuro.2011.01.014.

Jacome, Daniel E. and Robert J. Gumnit. 1979. "Audioalgesic and audiovisuoalgesic synesthesia: epileptic manifestation." Neurology 29: 1050-1053.

Jardri, Renaud, Delphine Pins, Maxime Bubrovszky, Bernard Lucas, Vianney Lethuc, Christine Delmaire, Vincent Vantyghem, Pascal Despretz and Pierre Thomas. 2009. "Neural functional organization of hallucinations in schizophrenia: multisensory dissolution of pathological emergence in consciousness." Consciousness and Cognition 18:449-457.

Jürgens, Uta Maria and Nikolić, Danko. 2012. "Ideaesthesia: Conceptual processes assign similar colours to similar shapes." Translational Neuroscience 3: 22-27. 
Kafka, J. S. 1997. "Romantic and classic visions in therapy of psychosis: A personal perspective and evolving theory of schizophrenia." Psychiatry 60: 262-271.

Keith, Allan. 2009. "The connotations of English colour terms: Colour-based X-phemisms." Journal of Pragmatics 41: 626-637.

Luke, David. 2011. "Discarnate entities and dimethyltryptamine (DMT): Psychopharmacology, phenomenology and ontology." Journal of the Society for Psychical Research 75: 26-42.

Makioka, Shogo A. 2009. Self-organizing learning account of number-form synaesthesia. Cognition 112: 397-414.

Marks, Lawrence E. 1975. "On colored-hearing synesthesia: Cross-modal translations of sensory dimensions." Psychological Bulletin 82: 303-331.

Marks, Lawrence E. 1989. "On cross-modal similarity. The perceptual structure of pitch, loudness, and brightness." Journal of Experimental Psychology: Human Perception and Performance 15: 589-602.

Marks, Lawrence E., Robin J. Hammeal and Marc H. Bornstein. 1987. "Perceiving similarity and comprehending metaphor." Monographs of the Society for Research in Child Development 52 (1, Serial No. 212).

Martino, Gail and Lawrence E. Marks. 2001. "Synesthesia: Strong and weak forms. Current Directions in Psychological Science 10: 61-65.

Maurer, Daphne. 1997. Neonatal synaesthesia: Implications for the processing of speech and faces. In Simon Baron-Cohen and John E. Harrison (eds.), Synaesthesia. Classic and contemporary readings, 224-242. Oxford: Blackwell.

Maurer, Daphne and Catherine J. Mondloch. 2005. Neonatal synesthesia: A reevaluation. In Lynn C. Robertson and Noam Sagiv (eds.), Synesthesia; Perspectives from cognitive neuroscience, 193-213. New York: Oxford University Press.

Meunier, David, Renaud Lambiotte and Edward T. Bullmore. 2010. "Modular and hierarchically modular organization of brain networks." Frontiers in Neuroscience 4: 1-11.

Mon-Williams, Mark, John P. Wann, Morgan Jenkinson and Kate Rushton. 1997. "Synaesthesia in the normal limb." Proceedings of the Royal Society of London, B 264: 1007-1010.

Mroczko, Aleksandra, Thomas Metzinger, Wolf Singer and Danko Nikolić. 2009. "Immediate transfer of synesthesia to a novel inducer." Journal of Vision 9: $1-8$.

Mulvenna, Catherine M. and Vincent Walsh. 2006. "Synaesthesia: supernormal integration?" Trends in Cognitive Sciences 10: 350-352.

Niccolai, Valentina, Tessa M. van Leeuwen, Colin Blakemore and Petra Stoerig. 2012. "Synaesthetic perception of colour and visual space in a blind subject: An fMRI case study." Consciousness and Cognition 21: 889-899.

Nikolić, Danko. 2009 (April). "Is synaesthesia actually ideaestesia? An inquiry into the nature of the phenomenon." Proceedings of the Third International Congress on Synaesthesia, Science \& Art, Granada, Spain. Retrieved from http://www. 
danko-nikolic.com/wp-content/uploads/2011/09/Synesthesia2009-NikolicIdeaesthesia.pdf

Nold, M. G. 1997. "Synesthesia and blindness: A personal account and informal survey." Journal of Visual Impairment \& Blindness 91: 14-15.

Novich, Scott, Sherry Cheng and David M. Eagleman. 2011. "Is synaesthesia one condition or many? A large-scale analysis reveals subgroups." Journal of Neuropsychology 5: 353-371.

Piaget, Jean. 1952. The origins of intelligence in children. New York: International Universities Press.

Podoll, Klaus and Derek Robinson. 2002. "Auditory-visual synaesthesia in patient with basilar migraine." Journal of Neurology, 249: 476-477.

Proulx, Michael J. 2010. "Synthetic synaesthesia and sensory substitution." Consciousness and Cognition 19: 501-503.

Proulx, Michael J. and Petra Stoerig. 2006. "Seeing sounds and tingling tongues: qualia in synaesthesia and sensory substitution." Anthropology \& Philosophy 7: $135-150$.

Proulx, Michael J., David J. Brown, Achille Pasqualotto and Peter Meijer. (in press). "Multisensory perceptual learning and sensory substitution." Neuroscience and Biobehavioral Reviews.

Pulvermüller, Friedemann. 1999. "Words in the brain's language." Behavioral and Brain Science 22: 253-336.

Pulvermüller, Friedemann. 2001. "Brain reflections of words and their meaning." Trends in Cognitive Sciences 5: 517-524.

Ramachandran, Vilayanur S. and Edward M. Hubbard. 2001. "Synaesthesia A window into perception, thought and language." Journal of Consciousness Studies 8: 3-34.

Ramachandran, Vilayanur S. and Diane Rogers-Ramachandran. 1996. "Synaesthesia in phantom limbs induced with mirrors." Proceedings of the Royal Society of London, B, 263: 377-386.

Ramachandran, Vilayanur S. and Diane Rogers-Ramachandran. 2000. "Phantom limbs and neural plasticity." Archives Neurology 57: 317-320.

Ramachandran, Vilayanur S., Diane Rogers-Ramachandran and S. Cobb. 1995. "Touching the phantom limb." Nature 377: 489-490.

Rao, Anling, Anna C. Nobre, Iona Alexander and Alan Cowey. 2007. "Auditory evoked visual awareness following sudden ocular blindness: An EEG and TMS investigation." Experimental Brain Research 176: 288-298.

Rich, Anina N., John L. Bradshaw and Jason B. Mattingley. 2005. "A systematic large scale study of synaesthesia: Implications for the role of early experience in lexical-colour associations." Cognition 98: 53-84.

Ro, Tony, Alessandro Farnè, Ruth M. Johnson, Van Wedeen, Zili Chu, Zhiyue J. Wang, Jill V. Hunter and Michael S. Beauchamp. 2007. "Feeling sounds after a thalamic lesion.” Annals of Neurology 62: 433-441. 
Ro, Tony, Johanan Hsu, Nafi E. Yasar, L. Caitlin Elmore and Michael S. Beauchamp. 2009. "Sound enhances touch perception." Experimental Brain Research 195: $135-143$.

Rogowska, Aleksandra. 2007. Synestezja [Synaesthesia]. Opole: Oficyna Wydawnicza PO.

Rogowska, Aleksandra. 2011. "Categorization of synaesthesia." Review of General Psychology 15: 213-227.

Rogowska, Aleksandra. 2014. Synaesthesia and individual differences. Manuscript submitted for publication.

Sarhan, Anwar, Haitham Jahrmi and Michael T. Compton. 2008. "A potential novel paradigm for testing digit-color synesthetic-like experiences in schizophrenia." Schizophrenia Research 98: 329-330.

Saugstad, Letten F. 2003. "Our neglect of the normal variation is linked to a reluctance to accept multifactorial inheritance and the role of environment. Medical Hypothese 60(2): 181-187.

Schaefer, Michael, Nina Noennig, Hans-Jochen Heinze and Michael Rotte. 2006. "Fooling yours feelings: Artificially induced referred sensations are linked to a modulation of the primary somatosensory cortex." NeuroImage 29: 67-73.

Seitz, Jay A. 2005. "The neural, evolutionary, developmental, and bodily basis of metaphor." New Ideas in Psychology 23: 74-95.

Shanon, Benny. 2003. "Three stories concerning synaesthesia: A commentary on Ramachandran and Hubbard." Journal of Consciousness Studies 10: 69-74.

Simner, Julia, Catherine Mulvenna, Noam Sagiv, Elias Tsakanikos, Sarah A. Witherby, Christine Fraser, Kirsten Scott and Jamie Ward. 2006. "Synaesthesia: the prevalence of atypical cross-modal experiences. Perception 35: 1024-1033.

Simner, Julia, Jamie Ward, Monika Lanz, Ashok Jansari, Krist Noonan, Louise Glover and David A. Oakley. 2005. "Non-random associations of graphemes to colours in synaesthetic and non-synaesthetic populations." Cognitive Neuropsychology 22: 1069-1085.

Sinke, Christopher, John H. Halpern, Markus Zedler, Janina Neufeld, Hinderk M. Emrich and Torsten Passie. 2012. "Genuine and drug-induced synesthesia: A comparison." Consciousness and Cognition 21: 1419-1434.

Spence, Charles. 2011. "Crossmodal correspondences: A tutorial review." Attention, Perception \& Psychophysics 73: 971-995.

Strauss, Milton E. 1993. "Relations of symptoms to cognitive deficits in schizophrenia." Schizophrenia Bulletin 19.2: 215-231.

Steen, Carol. 2001. "Visions shared: A firsthand look into synesthesia and art." Leonardo 34: 203-208.

Steven, Megan S., Peter C. Hansen and Colin Blakemore. 2006. Activation of colorselective areas of the visual cortex in a blind synesthete. Cortex 42: 304-308.

Studerus, Erich, Alex Gamma, Michael Kometer and Franz X. Vollenweider. 2012. "Prediction of psilocybin response in healthy volunteers." PLOS ONE 7.2: e30800. DOI:10.1371/journal.pone.003080. 
Studerus, Erich, Alex Gamma and Franz X. Vollenweider. 2010. Psychometric evaluation of the Altered States of Consciousness Rating Scale (OAV). PLoS ONE 5.8: e12412. DOI:10.1371/journal.pone.0012412.

Terhune, Devin B. 2009. "The incidence and determinants of visual phenomenology during out-of-body experiences." Cortex 45: 236-242.

Terhune, Devin B., Etzel Cardeña and Magnus Lindgren. 2010. "Disruption of synaesthesia by posthypnotic suggestion: An ERP study." Neuropsychologia 48: 3360-3364.

Thalbourne, Michael A., James Houran, A. G. Alias and Peter Brugger. 2001. "Transliminality, brain function, and synesthesia." Journal of Nervous and Mental Disease 189: 190-192.

Thornton-Wells, Tricia A., Chris J. Cannistraci, Adam Anderson, Chai-YounKim, Mariam Eapen, John C. Gore, Randolph Blake and Elisabeth M.Dykens. 2010. "Auditory Attraction: Activation of visual cortex by music and sound in Williams syndrome." American Journal on Intellectual and Developmental Disabilities 115: 172-189. DOI:10.1352/1944-7588-115.172.]

van Leeuwen, Tessa M., Hanneke E. M. den Ouden and Peter Hagoort. 2011. "Effective connectivity determines the nature of subjective experience in grapheme-color synesthesia." Journal of Neuroscience 31: 9879-9884. doi:10.1523/JNEUROSCI.0569-11.2011.

van Leeuwen, T. M. van, Petersson, K. M. and Hagoort, P. (2010b). Synaesthetic colour in the brain: beyond colour areas. A functional magnetic resonance imaging study of synaesthetes and matched controls. PLOS ONE 5: e12074.

Villemure, Chantal, Spogmai Wassimi, Gary J. Bennett, Yoram Shir and M. Catherine Bushnell. 2006. "Unpleasant odors increase pain processing in a patient with neuropathic pain: Psychophysical and fMRI investigation." Pain 120: 213220.

Walsh, Roger. 2005. "Can synaesthesia be cultivated?: Indications from surveys of meditators." Journal of Consciousness Studies 12: 5-17.

Ward, Jamie. 2004. "Emotionally mediated synaesthesia." Cognitive Neuropsychology 21: 761-772.

Ward, Jamie. 2007. "Acquired auditory-tactile synesthesia." Annals of Neurology 62: 429-430. DOI:10.1002/ana.21281

Ward, Jamie, Brett Huckstep and Elias Tsakanikos. 2006. "Sound-colour synaesthesia: to what extent does it use cross-modal mechanisms common to us all?" Cortex 42: 264-280.

Ward, Jamie, Ryan Li, Shireen Salih and Noam Sagiv. 2007. "Varieties of graphemecolour synaesthesia: A new theory of phenomenological and behavioural differences." Consciousness and Cognition 16: 913-931.

Ward, Jamie and Peter Meijer. 2010. "Visual experiences in the blind induced by an auditory sensory substitution device." Consciousness and Cognition 19: 492-500. 
Watson, Marcus R., Kathleen A. Akins and James T. Enns. 2012. "Second-order mappings in grapheme-color synesthesia." Psychonomic Bulletin and Review 19: 211-217.

Witthoft, Nathan and Jonathan Winawer. 2013. "Learning, memory, and synesthesia." Psychological Science 24(3): 258-265. DOI: 10.1177/0956797612452573

Witthoft, Nathan and Jonathan Winawer.2006. "Synesthetic colors determined by having colored refrigerator magnets in childhood." Cortex 42: 175-183.

Yaro, Caroline and Jamie Ward. 2007. "Searching for Shereshevskii: What is superior about the memory of synaesthetes?" The Quarterly Journal of Experimental Psychology 60: 681-695.

Zingrone, Nancy L., Carlos S. Alvarado and Natasha Agee. 2009. "Psychological correlates of aura vision: Psychic experiences, dissociation, absorption, and synaesthesia-like experiences." Australian Journal of Clinical and Experimental Hypnosis 37: 131-168. 